\title{
Síndrome compartimental abdominal
}

\section{Abdominal compartment syndrome}

Ana Isabel Sáez-Sáez, MD,,$^{* *}$ (1) Enrique De La Fuente-Fernández, MD, ${ }^{2}$ Luis Vicente Sáenz-Casco, MD, ${ }^{3}$

María Asunción Ramos-Meca, MD. ${ }^{4}$

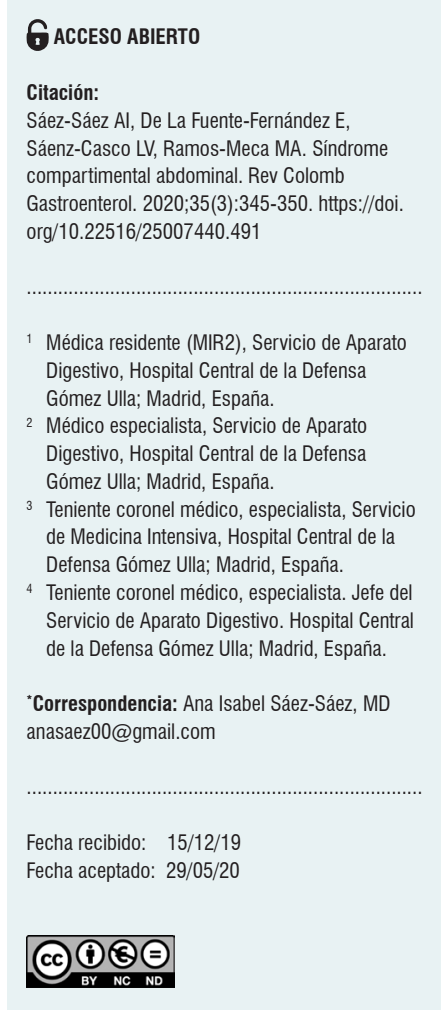

\section{Resumen}

El síndrome compartimental abdominal (SCA) se define como la disfunción orgánica causada por la hipertensión intraabdominal (HIA). Ambas son complicaciones frecuentes en pacientes graves ingresados en las unidades de cuidados intensivos (UCl). Las manifestaciones clínicas asociadas suelen ser inespecíficas, por lo que dichas entidades deben ser sospechadas ante pacientes críticos con factores de riesgo. El diagnóstico de la HIA se realiza midiendo de forma indirecta la presión intraabdominal (PIA), generalmente por medio de una sonda vesical. El tratamiento consiste en medidas de soporte, y en algunos casos es necesaria la descompresión abdominal quirúrgica. En esta revisión se presenta la fisiopatología de ambas entidades, así como el enfoque diagnóstico y terapéutico.

\section{Palabras clave}

Hipertensión intraabdominal, síndrome compartimental abdominal, pancreatitis aguda.

\section{Abstract}

Abdominal compartment syndrome (ACS) is defined as an organ dysfunction caused by intra-abdominal hypertension (IAH). Both are common complications in severe patients admitted to intensive care units. The associated clinical manifestations are usually non-specific and, therefore, such entities should be suspected in critical patients with risk factors. The diagnosis of IAH is made by indirectly measuring intrabdominal pressure, usually by means of a urinary catheter. Treatment consists of supportive measures and, in some cases, surgical abdominal decompression. In this review the physiopathology of both entities is described, as well as the diagnostic and therapeutic approach.

\section{Keywords}

Intra-abdominal hypertension; Abdominal compartment syndrome; Acute pancreatitis.

\section{INTRODUCCIÓN}

La hipertensión intraabdominal (HIA) y el síndrome compartimental abdominal (SCA) son complicaciones frecuentes en pacientes graves ingresados en las unidades de cuidados intensivos (UCI) (1). La investigación sobre ambas patologías en el último siglo ha permitido una mejoría diagnóstica, así como el desarrollo de medidas de prevención y tratamiento.

El último consenso de la World Society for Abdominal Compartment Syndrome (WSACS) (2) permitió establecer las definiciones de estas patologías e instaurar un protocolo 
diagnóstico y terapéutico basado en la evidencia científica. Así, la HIA se define como la presión intraabdominal (PIA) $\geq 12 \mathrm{~mm} \mathrm{Hg}$ y se puede clasificar según su duración:

- Hiperaguda: cuando dura segundos; por ejemplo, al reír, toser o estornudar.

- Aguda: aquella que se mantiene durante horas, como en casos de un traumatismo abdominal o una hemorragia intraabdominal.

- Subaguda: se desarrolla en días, como en el caso de la pancreatitis aguda grave o el choque séptico.

- Crónica: aquella que dura meses (como un embarazo) o años (como la obesidad mórbida). Estas no causan SCA, pero pueden agravarlo cuando se superpone una de las causas agudas o subagudas (1).

Entre tanto, el SCA es la disfunción orgánica causada por HIA y se define como una PIA $>20 \mathrm{~mm} \mathrm{Hg}$, asociada a una falla orgánica o a una disfunción de órganos que no estaba presente de forma previa. No está claro si el SCA es un fenómeno que deriva en una falla multiorgánica (FMO) o un epifenómeno que ocurre junto con otra disfunción de órgano.

El SCA se clasifica en primario, secundario y recurrente. El primario es aquel que se produce por una enfermedad en la región abdominopélvica (como en el caso de una pancreatitis aguda) y el secundario ocurre por condiciones no originadas a nivel abdominal, como, por ejemplo, una fluidoterapia agresiva en el choque séptico (2). Asimismo, el SCA recurrente se genera luego de un cierre de la pared abdominal o después del tratamiento de un SCA primario o secundario. En ocasiones puede existir un solapamiento entre varias entidades, lo que conduce a una mayor dificultad diagnóstica y terapéutica (3).

Otro concepto importante es la presión de perfusión abdominal (PPA), que se define como la diferencia entre la presión arterial media (PAM) y la PIA. Se ha demostrado que una PPA >60 mm Hg se correlaciona con una mejor supervivencia de HIA y SCA, así como con una menor probabilidad de desarrollo de una FMO (1-3).

Los pacientes con una PIA $<10 \mathrm{~mm} \mathrm{Hg}$ no suelen desarrollar SCA, a diferencia de aquellos con una PIA $>25 \mathrm{~mm}$ $\mathrm{Hg}$. Las personas que tienen una PIA entre 10 y $25 \mathrm{~mm} \mathrm{Hg}$ pueden o no desarrollar SCA, según variables individuales como la PPA y la distensibilidad de la pared abdominal. De igual forma, los pacientes que mantienen una correcta PPA (a pesar de tener una PIA elevada) tienen un menor riesgo de sufrir un SCA (4).

Por otro lado, la distensibilidad de la pared abdominal minimiza la medida a la cual el incremento de volumen intraabdominal puede elevar la PIA (1). La mortalidad del SCA se sitúa entre un 40 y un $100 \%$ (2-4). Las tasas de incidencia de HIA y SCA son muy variables según los criterios diagnósticos utilizados y la patología causante del cuadro.
En efecto, estudios recientes muestran una elevada prevalencia de estas enfermedades en pacientes médico-quirúrgicos ingresados en las UCI. Se estima una prevalencia en torno al $37-94 \%$ para la HIA y del 4-12 \% para el SCA.

\section{FACTORES DE RIESGO}

Las personas que desarrollan un SCA suelen ser pacientes críticos que son incapaces de comunicarse, cuyo manejo corresponde fundamentalmente a las UCI. Sus manifestaciones clínicas son inespecíficas, y entre ellas se incluyen malestar, debilidad, aturdimiento, disnea o dolor abdominal. La distensión abdominal es un mal predictor del SCA (3).

Con ese panorama, existen múltiples factores para el desarrollo del SCA. Estos se pueden clasificar según se produzca una disminución de la distensibilidad de la pared abdominal o del diafragma, así como un incremento del volumen intraabdominal o ambas (Tabla 1).

El choque séptico presenta una elevada prevalencia de HIA y de SCA, que se sitúa cerca del 82 y el $38 \%$, respectivamente. Por tanto, constituye un factor de riesgo, ya que se produce un choque distributivo que deriva en la vasodilatación periférica e incrementa la acumulación de fluidos en el tercer espacio. Todo esto se agrava por una resucitación masiva de fluidos (3-6).

En la pancreatitis aguda se produce una alteración similar, y se estima una incidencia de HIA de un $60 \%$ y de SCA de un $27 \%$, con una mayor prevalencia en las pancreatitis agudas graves (7). Se ha observado que la pancreatitis aguda asociada a HIA o SCA se relaciona con un incremento significativo en la extensión de la necrosis pancreática, FMO, tiempo de hospitalización y mortalidad.

Durante una revisión de 2014, que incluyó 7 estudios con un total de 271 pacientes con pancreatitis aguda, se encontró que aquellos enfermos con SCA tuvieron una mortalidad del $49 \%$, mientras que en quienes permanecieron sin SCA la mortalidad disminuyó al $11 \%$ (8). El incremento de la PIA se produce por la inflamación de los tejidos, la presencia de ascitis, la resucitación con fluidoterapia agresiva más allá de las 48 h, la existencia del íleo paralítico o la formación de colecciones pancreáticas. Aunque la reanimación con líquidos sigue siendo la piedra angular en el manejo de la pancreatitis aguda, la hidratación excesiva más allá de las $48 \mathrm{~h}$ puede aumentar la mortalidad (8).

Los pacientes obesos tienen un mayor riesgo de sufrir una pancreatitis aguda grave $(9,10)$. En este sentido, se han implicado múltiples factores locales y sistémicos en la patogénesis del pronóstico adverso que supone la obesidad. A nivel sistémico, la obesidad en sí misma constituye un estado proinflamatorio, mientras que a nivel local se ha demostrado que los depósitos grasos peripancreáticos en personas obesas producen una necrosis grasa más extensa. 
Tabla 1. Factores de riesgo para el desarrollo del SCA

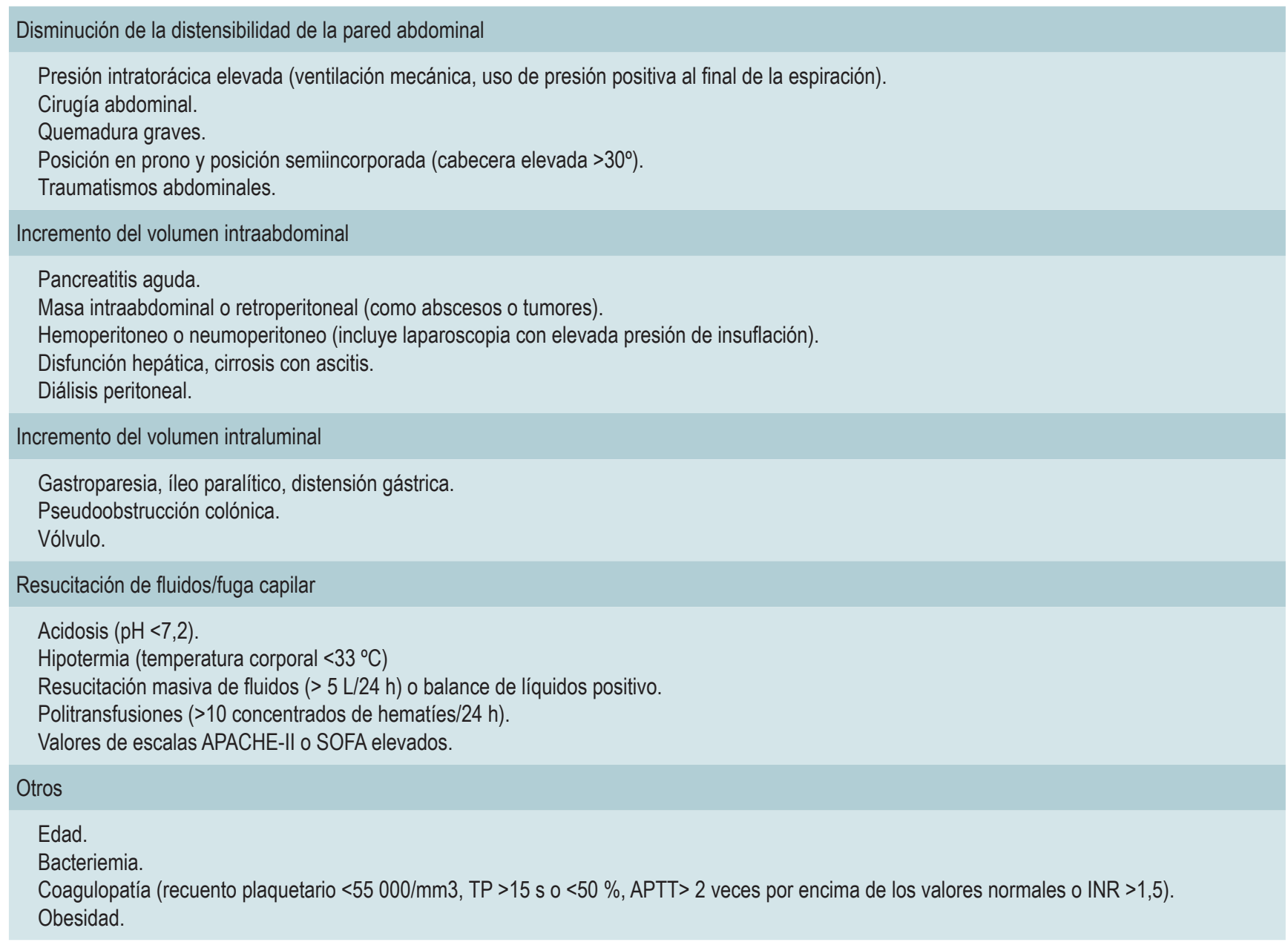

SCA: síndrome compartimental abdominal; TP: tiempo de protrombina; APTT: tiempo de tromboplastina parcial activado (Activated Partial ThromboplastinTime); INR: índice internacional normalizado (International Normalized Ratio); APACHE II: evaluación de la salud crónica y la fisiología aguda (Acute Physiology And Chronic Health Evaluation II); SOFA: evaluación de falla orgánica secuencial (Sequential Organ Failure Assessment). Modificada de las referencias 2 y 3 .

Así pues, esta condición supone un factor de riesgo para desarrollar SCA. No obstante, existen pocos estudios que relacionen la obesidad en la pancreatitis aguda con el SCA.

\section{FISIOPATOLOGÍA}

El aumento de la PIA puede alterar la función de diferentes órganos y sistemas. A nivel del sistema nervioso central (SNC), este incremento puede generar una elevación de la presión intracraneal, con una reducción de la presión de perfusión cerebral, ya que afecta el retorno venoso. A nivel cardiovascular, se produce un aumento de la presión venosa central y de la presión capilar pulmonar, así como una disminución del retorno venoso, un incremento de las resistencias vasculares y una reducción del gasto cardíaco.

Por otro lado, el incremento de la PIA causa un aumento de las presiones de la vía aérea y de la presión intratorácica, y una reducción de la distensibilidad total del sistema respiratorio, sobre todo, de la pared torácica por compresión diafragmática. Entonces, se produce un incremento del espacio muerto y del shunt intrapulmonar, lo que conduce al desarrollo de atelectasias. Todo esto crea una tendencia hacia la hipercapnia y la hipoxemia (1-3).

Con respecto a los efectos a nivel gastrointestinal, si la PIA es $>20 \mathrm{~mm} \mathrm{Hg}$, se produce una disminución de la perfusión de la mucosa intestinal, mientras que si la PIA es $>40 \mathrm{~mm}$ 
$\mathrm{Hg}$, ocurre una reducción de la perfusión de la arteria celíaca y mesentérica superior. Entre tanto, la disminución del flujo sanguíneo en la mucosa intestinal deriva en un descenso del $\mathrm{pH}$ intramucoso gástrico y en una isquemia posterior.

A nivel hepático, se da una disminución del aclaramiento del lactato, así como una reducción en el resto de sus funciones metabólicas, con la posibilidad de que ocurra una elevación de las enzimas hepáticas por hipoxia hepatocelular y lesión centrolobulillar.

Asimismo, el riñón es uno de los órganos de mayor vulnerabilidad y la oliguria constituye uno de los signos más precoces. Esta se produce por una disminución del filtrado glomerular y una activación del sistema renina-angiotensina-aldosterona. La oliguria es frecuente si la HIA es $>15$ $\mathrm{mm} \mathrm{Hg}$, mientras que la anuria ocurre si la HIA es $>30$ $\mathrm{mm} \mathrm{Hg}$ (1-3).

\section{DIAGNÓSTICO}

Para realizar el diagnóstico de HIA se debe medir la PIA de forma indirecta, mediante una sonda vesical, intragástrica o intracolónica, o por medio de la inserción de un catéter en la vena cava inferior. La sonda vesical es la recomendada por la WSACS (2), ya que es simple, mínimamente invasiva, precisa y económica. Para una adecuada medición, el paciente debe estar posicionado en decúbito supino, con la cama a $0^{\circ}$.

La PIA se mide al final de la espiración, sin contracciones abdominales voluntarias y posicionando la sonda a nivel de la línea media axilar, en la cresta ilíaca. En caso de HIA, se recomienda medir la PIA cada 4-6 h, o incluso, en ciertas circunstancias, de manera continua $(1,2,5)$.

\section{TRATAMIENTO}

El tratamiento inicial es conservador y escalonado (2). Existe una serie de medidas no quirúrgicas para la prevención y el manejo de estas entidades, que se basan en cuatro pilares fundamentales $(2,3)$ : a) disminuir o evacuar el contenido intraluminal, mediante la inserción de una sonda nasogástrica o rectal; iniciar con agentes procinéticos; disminuir o suspender la nutrición enteral; administrar enemas, y realizar la descompresión por medio de una colonoscopia; b) evaluar el drenaje de las lesiones ocupantes de espacio a nivel abdominal, mediante un catéter percutáneo o una cirugía; c) mejorar la distensibilidad de la pared abdominal, asegurando una adecuada sedación $\mathrm{y}$ analgesia; valorar la posición de anti-Trendelemburg, y como última opción, considerar el bloqueo neuromuscular; d) optimizar la resucitación con fluidoterapia y evitar una sueroterapia agresiva, para lo cual se debe efectuar un balance hídrico neutro o incluso negativo. Otras medidas, como usar coloides o la hemodiálisis, están en controversia en la actualidad.

La prevención e identificación precoz de los factores de riesgo es la mejor forma de actuar frente al SCA. Cuando el SCA está establecido, la descompresión quirúrgica es el tratamiento de elección. No se ha establecido un umbral preciso para realizar la descompresión quirúrgica, ni se ha determinado qué técnica sería la más adecuada. En caso de disfunción orgánica, algunos autores recomiendan la descompresión quirúrgica cuando la PIA es $>25 \mathrm{~mm} \mathrm{Hg}$ (11-14).

Otros autores, en cambio, recomiendan que la descompresión quirúrgica esté determinada por la PPA, la cual es de mal pronóstico cuando es $<50-60 \mathrm{~mm} \mathrm{Hg}$. En aquellos pacientes que, a pesar del tratamiento médico, continúan con PIA elevadas asociadas a una FMO, se debe considerar la laparotomía descompresiva. Tras resolver la patología intraabdominal, se puede realizar un cierre de la cavidad abdominal sin tensión o dejar un abdomen abierto.

En el abdomen abierto, la pared abdominal se puede cerrar mediante diferentes métodos $(2,3)$ : a) el cierre por medio de pinzas o grapas para aproximar la piel; b) la cobertura mediante una bolsa translúcida esterilizada denominada bolsa de Bogotá; c) el cierre con un parche de Wittmann o el cierre dinámico tipo ABRA (Abdominal reaproximation Anchor System); d) la técnica sándwich; e) el cierre con materiales protésicos o coberturas siliconadas; f) las técnicas de cierre con terapia de presión negativa.

Las más utilizadas hoy en día son las técnicas que usan presión negativa. El cierre asistido por aspiración, también llamado VAC (Vacuum-Assisted Closure), aplica una presión negativa que favorece la retracción de la musculatura abdominal y permite el cierre diferido de la fascia. Se produce, entonces, una absorción y la evacuación del contenido líquido abdominal, lo que facilita el control de la PIA.

Entre tanto, la laparotomía descompresiva genera una mejoría inmediata en el descenso de la PIA y mejora la disfunción orgánica, sin excluir complicaciones que pueden llegar hasta el $50 \%$ (2). Cuanto más tiempo esté el abdomen abierto, mayor será la morbilidad. Por tanto, se deben considerar estrategias específicas para prevenir las adherencias viscerales, la pérdida de cobertura de tejidos blandos, la lateralización de la musculatura abdominal y su fascia, la desnutrición y las fístulas entéricas (2).

Los sistemas de presión negativa son de elección en la terapia de heridas abdominales (2), ya que disminuyen la incidencia de complicaciones quirúrgicas como la infección de la herida, eventraciones por la retracción de la fascia abdominal, hernias o alteraciones hidroelectrolíticas. Como complicación, estas estrategias presentan la aparición de fístulas enterocutáneas o enteroatmosféricas, 
con una incidencia que ronda el $20 \%$. Dicha complicación puede ocurrir incluso a los 8 días de la laparotomía inicial. Los pacientes con una anastomosis intestinal tienen el mayor riesgo de sufrir esta complicación (2).

Respecto a la cirugía de control de daños, la WSACS (2) sugiere que los pacientes sometidos a una laparotomía abdominal, por causa de un traumatismo que sufren por agotamiento fisiológico, podrían ser tratados con el uso profiláctico del abdomen abierto frente al cierre y control de la HIA.
Para pacientes agudos no traumáticos, existe muy poca evidencia que respalde las técnicas de control de daños con el uso profiláctico del abdomen abierto. En enfermos con sepsis abdominal, sometidos a una laparotomía de urgencia, los autores recomiendan no utilizar de forma rutinaria el abordaje abierto abdominal, salvo que la HIA sea una preocupación específica.

\section{REFERENCIAS}

1. De Waele JJ, Cheatham ML, Malbrain ML, Kirkpatrick AW, Sugrue M, Balogh Z, Ivatury R, De Keulenaer B, Kimball EJ. Recommendations for research from the International Conference of Experts on Intra-abdominal Hypertension and Abdominal Compartment Syndrome. Acta Clin Belg. 2009;64(3):203-9.

http://doi.org/10.1179/acb.2009.036

2. Kirkpatrick AW, Roberts DJ, De Waele J, Jaeschke R, Malbrain ML, De Keulenaer B, Duchesne J, Bjorck M, Leppaniemi A, Ejike JC, Sugrue M, Cheatham M, Ivatury R, Ball CG, Reintam Blaser A, Regli A, Balogh ZJ, D’Amours S, Debergh D, Kaplan M, Kimball E, Olvera C; Pediatric Guidelines Sub-Committee for the World Society of the Abdominal Compartment Syndrome. Intra-abdominal hypertension and the abdominal compartment syndrome: updated consensus definitions and clinical practice guidelines from the World Society of the Abdominal Compartment Syndrome. Intensive Care Med. 2013;39(7):1190-206. http://doi.org/10.1007/s00134-013-2906-z

3. Sánchez-Miralles A, Castellanos G, Badenes R, Conejero R. Síndrome compartimental abdominal y síndrome de distrés intestinal agudo. Med Intensiva. 2013;37(2):99-109. http://doi.org/10.1016/j.medin.2011.11.019

4. Malbrain ML, Chiumello D, Pelosi P, Bihari D, Innes R, Ranieri VM, Del Turco M, Wilmer A, Brienza N, Malcangi V, Cohen J, Japiassu A, De Keulenaer BL, Daelemans R, Jacquet L, Laterre PF, Frank G, de Souza P, Cesana B, Gattinoni $\mathrm{L}$. Incidence and prognosis of intraabdominal hypertension in a mixed population of critically ill patients: a multiple-center epidemiological study. Crit Care Med. 2005;33(2):315-22.

http://doi.org/10.1097/01.ccm.0000153408.09806.1b

5. Malbrain ML. Different techniques to measure intraabdominal pressure (IAP): time for a critical re-appraisal. Intensive Care Med. 2004;30(3):357-371. http://doi.org/10.1007/s00134-003-2107-2
6. Wilson A, Longhi J, Goldman C, McNatt S. Intraabdominal pressure and the morbidly obese patients: the effect of body mass index. J Trauma. 2010;69(1):78-83. http://doi.org/10.1097/TA.0b013e3181e05a79

7. Trikudanathan G, Vege SS. Current concepts of the role of abdominal compartment syndrome in acute pancreatitis - an opportunity or merely an epiphenomenon. Pancreatology. 2014;14(4):238-243. http://doi.org/10.1016/j.pan.2014.06.002

8. van Brunschot S, Schut AJ, Bouwense SA, Besselink MG, Bakker OJ, van Goor H, Hofker S, Gooszen HG, Boermeester MA, van Santvoort HC; Dutch Pancreatitis Study Group. Abdominal compartment syndrome in acute pancreatitis: a systematic review. Pancreas. 2014;43(5):665-74. http://doi.org/10.1097/MPA.0000000000000108

9. Cruz-Monserrate Z, Conwell DL, Krishna SG. The Impact of Obesity on Gallstone Disease, Acute Pancreatitis, and Pancreatic Cancer. Gastroenterol Clin North Am. 2016;45(4):625-637. http://doi.org/10.1016/j.gtc.2016.07.010

10. Davis PJ, Eltawil KM, Abu-Wasel B, Walsh MJ, Topp T, Molinari M. Effect of obesity and decompressive laparotomy on mortality in acute pancreatitis requiring intensive care unit admission. World J Surg. 2013;37(2):318-332. http://doi.org/10.1007/s00268-012-1821-8

11. Malbrain ML, Cheatham ML, Kirkpatrick A, Sugrue M, Parr M, De Waele J, Balogh Z, Leppäniemi A, Olvera C, Ivatury R, D’Amours S, Wendon J, Hillman K, Johansson $\mathrm{K}$, Kolkman K, Wilmer A. Results from the International Conference of Experts on Intra-abdominal Hypertension and Abdominal Compartment Syndrome. I. Definitions. Intensive Care Med. 2006;32(11):1722-32. http://doi.org/10.1007/s00134-006-0349-5

12. Cheatham ML, Malbrain ML, Kirkpatrick A, Sugrue M, Parr M, De Waele J, Balogh Z, Leppäniemi A, Olvera C, Ivatury R, D'Amours S, Wendon J, Hillman K, Wilmer 
A. Results from the International Conference of Experts on Intra-abdominal Hypertension and Abdominal

Compartment Syndrome. II. Recommendations. Intensive Care Med. 2007;33(6):951-62.

http://doi.org/10.1007/s00134-007-0592-4

13. De Waele JJ, De Laet I, Kirkpatrick AW, Hoste E. Intraabdominal Hypertension and Abdominal Compartment
Syndrome. Am J Kidney Dis. 2011;57(1):159-169.

http://doi.org/10.1053/j.ajkd.2010.08.034

14. Mentula P, Hienonen P, Kemppainen E, Puolakkainen P, Leppäniemi A. Surgical decompression for abdominal compartment syndrome in severe acute pancreatitis. Arch Surg. 2010;145(8):764-769.

http://doi.org/10.1001/archsurg.2010.132 